\title{
Descents of Permutations in a Ferrers Board
}

\author{
Chunwei Song* \\ School of Mathematical Sciences, LMAM, Peking University, \\ Beijing 100871, P. R. China \\ Catherine Yan $^{\dagger}$ \\ Department of Mathematics, Texas A\&M University, \\ College Station, TX 77843-3368
}

Submitted: Sep 6, 2011; Accepted: Dec 20, 2011; Published: Jan 6, 2012

Mathematics Subject Classification: 05A05, 05A15, 05A30

Abstract

The classical Eulerian polynomials are defined by setting

$$
A_{n}(t)=\sum_{\sigma \in \mathfrak{S}_{n}} t^{1+\operatorname{des}(\sigma)}=\sum_{k=1}^{n} A_{n, k} t^{k}
$$

where $A_{n, k}$ is the number of permutations of length $n$ with $k-1$ descents. Let $A_{n}(t, q)=\sum_{\pi \in \mathfrak{S}_{n}} t^{1+\operatorname{des}(\pi)} q^{\operatorname{inv}(\pi)}$ be the inv $q$-analogue of the classical Eulerian polynomials whose generating function is well known:

$$
\sum_{n \geqslant 0} \frac{u^{n} A_{n}(t, q)}{[n]_{q} !}=\frac{1}{1-t \sum_{k \geqslant 1} \frac{(1-t)^{k} u^{k}}{[k]_{q} !}} .
$$

In this paper we consider permutations restricted in a Ferrers board and study their descent polynomials. For a general Ferrers board $F$, we derive a formula in the form of permanent for the restricted $q$-Eulerian polynomial

$$
A_{F}(t, q):=\sum_{\sigma \in F} t^{1+\operatorname{des}(\sigma)} q^{\operatorname{inv}(\sigma)}
$$

If the Ferrers board has the special shape of an $n \times n$ square with a triangular board of size $s$ removed, we prove that $A_{F}(t, q)$ is a sum of $s+1$ terms, each satisfying an equation that is similar to (0.1). Then we apply our results to permutations with bounded drop (or excedance) size, for which the descent polynomial was first studied by Chung et al. (European J. Combin., 31(7) (2010): 1853-1867). Our method presents an alternative approach.

*Supported in part by NSF China grant \#10726011 and by the Scientific Research Foundation for ROCS, Chinese Ministry of Education.

${ }^{\dagger}$ Supported in part by NSF grant \#DMS-0653846 and NSA grant \#H98230-11-1-0167. 


\section{Introduction}

Let $\mathfrak{S}_{n}$ denote the symmetric group of order $n$. Given a permutation $\sigma \in \mathfrak{S}_{n}$, let Des $(\sigma)$ be the descent set of $\sigma$, i.e., $\operatorname{Des}(\sigma)=\left\{i \mid \sigma_{i}>\sigma_{i+1}, 1 \leqslant i \leqslant n-1\right\}$, and let $\operatorname{des}(\sigma)=|\operatorname{Des}(\sigma)|$ denote the number of descents of $\sigma$. For $D \subseteq\{1,2, \ldots, n-1\}$, we denote by $\alpha_{n}(D)$ the number of permutations $\pi \in \mathfrak{S}_{n}$ whose descent set is contained in $D$, and by $\beta_{n}(D)$ the number of permutations $\pi \in \mathfrak{S}_{n}$ whose descent set is equal to $D$. In symbols,

$$
\alpha_{n}(D):=\left|\left\{\sigma \in \mathfrak{S}_{n}: \operatorname{Des}(\sigma) \subseteq D\right\}\right|, \quad \beta_{n}(D):=\left|\left\{\sigma \in \mathfrak{S}_{n}: \operatorname{Des}(\sigma)=D\right\}\right| .
$$

Let $D=\left\{d_{1}, d_{2}, \ldots, d_{k}\right\}$ where $1 \leqslant d_{1}<\cdots<d_{k} \leqslant n-1$. For convenience, also let $d_{0}=0$ and $d_{k+1}=n$. Then the following formulas for $\alpha_{n}(D)$ and $\beta_{n}(D)$ are well-known (see, for example, [14, p.69]):

$$
\begin{aligned}
& \alpha_{n}(D)=\left(\begin{array}{c}
n \\
d_{1}, d_{2}-d_{1}, \ldots, n-d_{k}
\end{array}\right) \\
& \beta_{n}(D)=n ! \operatorname{det}\left[\frac{1}{\left(d_{j+1}-d_{i}\right) !}\right]=\operatorname{det}\left[\left(\begin{array}{c}
n-d_{i} \\
d_{j+1}-d_{i}
\end{array}\right)\right],
\end{aligned}
$$

where $(i, j) \in[0, k] \times[0, k]$ in the matrix of equation (1.2).

A $q$-analogue of the above formulas is given by considering the permutation statistic $\operatorname{inv}(\sigma)$, where $\operatorname{inv}(\sigma)=\sum_{i<j} \chi\left(\sigma_{i}>\sigma_{j}\right)$. By convention, the symbol $\chi(P)$ is 1 if the statement $P$ is true and 0 if not. See [14, Example 2.2.5]. Explicitly, let

$$
\alpha_{n}(D, q)=\sum_{\pi \in \mathfrak{S}_{n}: \operatorname{Des}(\pi) \subseteq D} q^{\operatorname{inv}(\pi)}, \quad \beta_{n}(D, q)=\sum_{\pi \in \mathfrak{S}_{n}: \operatorname{Des}(\pi)=D} q^{\operatorname{inv}(\pi)} .
$$

Then

$$
\begin{aligned}
& \alpha_{n}(D, q)=\left[\begin{array}{c}
n \\
d_{1}, d_{2}-d_{1}, \ldots, n-d_{k}
\end{array}\right]=\frac{[n] !}{\left[d_{1}\right] !\left[d_{2}-d_{1}\right] ! \cdots\left[n-d_{k}\right] !} \\
& \beta_{n}(D, q)=[n] ! \operatorname{det}\left[\frac{1}{\left[d_{j+1}-d_{i}\right] !}\right]=\operatorname{det}\left[\left[\begin{array}{c}
n-d_{i} \\
d_{j+1}-d_{i}
\end{array}\right]\right],
\end{aligned}
$$

where $(i, j) \in[0, k] \times[0, k]$ as before. Here we use the standard notation

$$
[n]:=\left(1-q^{n}\right) /(1-q), \quad[n] !:=[1][2] \cdots[n], \quad\left[\begin{array}{l}
n \\
k
\end{array}\right]:=\frac{[n]_{q} !}{[k] ![n-k] !}
$$

for the $q$-analogue of the integer $n$, the $q$-factorial, and the $q$-binomial coefficient, respectively. Sometimes it is necessary to write the base $q$ explicitly as in $[n]_{q},[n]_{q} !$, and $\left[\begin{array}{l}n \\ k\end{array}\right]_{q}$, etc., but we omit $q$ in this paper as we do not use the analogues of any other variables.

The classical Eulerian polynomials are defined by setting

$$
A_{n}(t)=\sum_{\sigma \in \mathfrak{S}_{n}} t^{1+\operatorname{des}(\sigma)}=\sum_{k=1}^{n} A_{n, k} t^{k}
$$


where $A_{n, k}$ is called the Eulerian number that denotes the number of permutations of length $n$ with $k-1$ descents. Let $A_{0}(t)=1$. The polynomials $A_{n}(t)$ have the generating function (see e.g. Riordan [12])

$$
\sum_{n \geqslant 0} A_{n}(t) \frac{u^{n}}{n !}=\frac{1}{1-t \sum_{k \geqslant 1} \frac{(1-t)^{k-1} u^{k}}{k !}}=\frac{1-t}{1-t e^{u(1-t)}}
$$

Let $A_{n}(t, q)=\sum_{\pi \in \mathfrak{S}_{n}} t^{1+\operatorname{des}(\pi)} q^{\operatorname{inv}(\pi)}$ be the inv $q$-analogue of the Eulerian polynomials. Stanley [13] showed that

$$
\sum_{n \geqslant 0} \frac{u^{n} A_{n}(t, q)}{[n] !}=\frac{1-t}{1-t E(u(1-t) ; q)}
$$

where

$$
E(z ; q)=\sum_{n \geqslant 0} \frac{z^{n}}{[n] !} .
$$

By simple manipulations we can see that an equivalent form of (1.6) is

$$
\sum_{n \geqslant 0} \frac{u^{n} A_{n}(t, q)}{[n] !}=\frac{1}{1-t \sum_{k \geqslant 1} \frac{(1-t)^{k-1} u^{k}}{[k] !}}
$$

Alternative proofs of (1.7) have been given by Gessel [9] and Garsia [8].

In this paper we consider permutations with restricted positions, and extend the above results to descent polynomials of permutations in a Ferrers board. Traditionally a permutation $\sigma \in \mathfrak{S}_{n}$ is also represented as a 01-filling of an $n$ by $n$ square board: Reading from left to right and bottom to top, we simply put a 1 in the $i$ th row and the $j$ th column whenever $\sigma_{i}=j$ for $i=1, \ldots, n$. Given integers $0<r_{1} \leqslant r_{2} \leqslant \cdots \leqslant r_{n}$, the Ferrers board of shape $\left(r_{1}, \ldots, r_{n}\right)$ is defined by

$$
F=\left\{(i, j): 1 \leqslant i \leqslant n, 1 \leqslant j \leqslant r_{i}\right\}
$$

In the following we identify a permutation $\sigma$ with its 01-filling representation, and say that $\sigma$ is in a Ferrers board $F$ if all the cells $\left(i, \sigma_{i}\right)$ are in $F$.

In Section 2 we extend the formulas (1.3) and (1.4) to the set of permutations on a fixed Ferrers shape with $n$ rows and $n$ columns, and derive a permanent formula for the restricted $q$-Eulerian polynomial

$$
A_{F}(t, q):=\sum_{\sigma \in F} t^{1+\operatorname{des}(\sigma)} q^{\operatorname{inv}(\sigma)} .
$$

In Section 3 we focus on the Ferrers board that is obtained from the $n \times n$ square by removing a triangular board of size $s$, and prove that the restricted $q$-Eularian polynomial is a sum of $s+1$ terms, each determined by an equation that generalizes (1.7). 
Finally in Section 4, we apply our results to permutations with bounded drop (or excedance) size, for which the descent polynomial was first studied by Chung, Claesson, Dukes and Graham [4]. Our method presents an alternative approach to the results in $[4]$.

\section{Notation on lattice path}

Here we recall some notation and results about the counting of lattice paths with a general right boundary. These results offer the main tool to describe permutations restricted in a Ferrers board. For a reference on lattice path counting, see Mohanty [11].

A lattice path $P$ is a path in the plane with two kinds of steps: a unit north step $N$ or a unit east step $E$. If $x$ is a positive integer, a lattice path from the origin $(0,0)$ to the point $(x, n)$ can be coded by a length $n$ non-decreasing sequence $\left(x_{1}, x_{2}, \ldots, x_{n}\right)$, where $0 \leqslant x_{i} \leqslant x$ and $x_{i}$ is the $x$-coordinate of the $i$ th north step. For example, let $x=5$ and $n=3$. Then the path EENENNEE is coded by $(2,3,3)$.

In general, let $\mathbf{s}$ be a non-decreasing sequence with positive integer terms $s_{1}, s_{2}, \ldots, s_{n}$. A lattice path from $(0,0)$ to $(x, n)$ is one with the right boundary $\mathbf{s}$ if $x_{i}<s_{i}$ for $1 \leqslant i \leqslant n$. If $x \geqslant s_{n}$, then the number of lattice paths from $(0,0)$ to $(x, n)$ with the right boundary $\mathbf{s}$ does not depend on $x$. Let $\operatorname{Path}_{n}(\mathbf{s})$ be the set of lattice paths from $(0,0)$ to $\left(s_{n}, n\right)$ with the right boundary s, and $L P_{n}(\mathbf{s})$ be the cardinality of $\operatorname{Path}_{n}(\mathbf{s})$. For a given sequence $\mathbf{s}=\left(s_{1}, s_{2}, \ldots, s_{n}\right)$, let

$$
L P_{n}(\mathbf{s} ; q)=\sum_{P \in \operatorname{Path}_{n}(\mathbf{s})} q^{\operatorname{area}(P)},
$$

where $\operatorname{area}(P)=\sum_{i=1}^{n} x_{i}$ is the area enclosed by the path $P$, the $y$-axis, and the line $y=n$. Hence $L P_{n}(\mathbf{s})=L P_{n}(\mathbf{s} ; 1)$. In this paper we will also allow the entries $s_{i}$ to satisfy $s_{1} \geqslant s_{2} \geqslant \cdots \geqslant s_{n}$, in which case

$$
L P_{n}(\mathbf{s} ; q)=L P_{n}\left(\left(s_{n}, s_{n}, \ldots, s_{n}\right) ; q\right)=\left[\begin{array}{c}
s_{n}+n-1 \\
n
\end{array}\right] .
$$

In particular $L P_{n}((n+1, n+1, \ldots, n+1) ; q)=\left[\begin{array}{c}2 n \\ n\end{array}\right]$. It is also easy to see that

$$
L P_{n}((1,2, \ldots, n) ; q)=C_{n}(q)
$$

where $C_{n}(q)$ is Carlitz-Riordan's $q$-Catalan number [2].

\section{Descents of permutations in Ferrers boards}

Let $F$ be a Ferrers board with $n$ rows and $n$ columns, which is aligned on the top and left. Index the rows from bottom to top, and columns from left to right. Let $r_{i}$ be the size of row $i$. Hence $1 \leqslant r_{1} \leqslant r_{2} \leqslant \cdots \leqslant r_{n}=n$.

For a set $D=\left\{d_{1}, d_{2}, \ldots, d_{k}\right\}$ with $1 \leqslant d_{1}<\cdots<d_{k} \leqslant n-1$, let $\beta_{F}(D)$ be the number of permutations in $F$ with the descent set $D$, and $\alpha_{F}(D)$ be the number of permutations 
in $F$ whose descent set is contained in $D$. The inv $q$-analogues of $\alpha_{F}(D)$ and $\beta_{F}(D)$ are defined by

$$
\alpha_{F}(D, q)=\sum_{\sigma \in F: \operatorname{Des}(\sigma) \subseteq D} q^{\operatorname{inv}(\sigma)}, \quad \beta_{F}(D, q)=\sum_{\sigma \in F: \operatorname{Des}(\sigma)=D} q^{\operatorname{inv}(\sigma)} .
$$

Clearly $\alpha_{F}(D, 1)=\alpha_{F}(D)$ and $\beta_{F}(D, 1)=\beta_{F}(D)$. The Inclusion-Exclusion Principle implies that

$$
\alpha_{F}(D, q)=\sum_{T \subseteq D} \beta_{F}(T, q), \quad \beta_{F}(D, q)=\sum_{T \subseteq D}(-1)^{|D-T|} \alpha_{F}(T, q)
$$

We shall show that $\alpha_{F}(D, q)$ and $\beta_{F}(D, q)$ can be expressed in terms of $L P_{n}(\mathbf{s}, q)$, the area enumerator of lattice paths with proper right boundaries and lengths.

Let's first compute $\alpha_{F}(D, q)$. To get a permutation $\sigma$ in $F$ satisfying $\operatorname{Des}(\sigma) \subseteq D$, we first choose $x_{1}<x_{2}<\cdots<x_{d_{1}}$ such that $1 \leqslant x_{i} \leqslant r_{i}$, and put a 1 in the cell $\left(x_{i}, i\right)$ for $1 \leqslant i \leqslant d_{1}$. Then choose $x_{d_{1}+1}<x_{d_{1}+2}<\cdots<x_{d_{2}}$ such that $1 \leqslant x_{i} \leqslant r_{i}$, and put a 1 in the cell $\left(x_{i}, i\right)$ for $d_{1}<i \leqslant d_{2}$, and so on.

We say that the cell $(i, j)$ is a 1-cell if it is filled with a 1 . It is clear that an inversion of $\sigma$ corresponds to a southeast chain of size 2 in the filling, i.e. a pair of 1-cells \{ $\left.\left(x_{i_{1}}, i_{1}\right),\left(x_{i_{2}}, i_{2}\right)\right\}$ such that $i_{1}<i_{2}$ while $x_{i_{1}}>x_{i_{2}}$.

For $1 \leqslant i \leqslant d_{1}$, the 1 -cell in the $i$ th row (i.e. $y=i$ ) has exactly $x_{i}-i$ many other 1-cells lying above it and to its left. Hence the 1-cell in the $i$ th row contributes $x_{i}-i$ to the statistic $\operatorname{inv}(\sigma)$, and all the 1-cells in the first $d_{1}$ rows contributed

$$
\left(x_{1}-1\right)+\left(x_{2}-2\right)+\cdots+\left(x_{d_{1}}-d_{1}\right)
$$

to the statistic $\operatorname{inv}(\sigma)$.

Note that $0 \leqslant x_{1}-1 \leqslant x_{2}-2 \leqslant \cdots \leqslant x_{d_{1}}-d_{1}$, and $x_{i}-i<r_{i}-i+1$. Hence the number of choices for the sequence $\left(x_{1}, \ldots, x_{d_{1}}\right)$ is exactly the number of lattice paths from $(0,0)$ to $\left(r_{d_{1}}-d_{1}+1, d_{1}\right)$ with the right boundary $\left(r_{1}, r_{2}-1, \ldots, r_{d_{1}}-d_{1}+1\right)$, and $\sum_{i=1}^{d_{1}}\left(x_{i}-i\right)$ is the area of the corresponding lattice path. Therefore the first $d_{1}$ rows of $F$ contribute a factor of $L P_{d_{1}}\left(\left(h_{1}, \ldots, h_{d_{1}}\right) ; q\right)$ to $\alpha_{F}(D, q)$.

Let $\mathbf{h}=\left(h_{1}, h_{2}, \ldots, h_{n}\right)$ where $h_{i}=r_{i}-i+1$. Let the $i$-th block of $F$ consist of rows $d_{i-1}+1$ to $d_{i}$. Applying the above analysis to the $i$-th block of the Ferrers board $F$ for $i=2, \ldots, k+1$, we get that

\section{Theorem 2.1}

$$
\alpha_{F}(D, q)=\sum_{\sigma \in F: \operatorname{Des}(\sigma) \subseteq D} q^{\operatorname{inv}(\sigma)}=\prod_{i=0}^{k} L P_{d_{i+1}-d_{i}}\left(\left(h_{d_{i}+1}, \ldots, h_{d_{i+1}}\right) ; q\right)
$$

where we use the convention that $d_{0}=0$ and $d_{k+1}=n$. 
Accordingly,

$$
\begin{aligned}
\beta_{F}(D, q) & =\sum_{T \subseteq D}(-1)^{|D-T|} \alpha_{F}(T, q) \\
& =\sum_{1 \leqslant i_{1}<i_{2}<\cdots<i_{j} \leqslant k}(-1)^{k-j} f\left(0, i_{1}\right) f\left(i_{1}, i_{2}\right) \ldots f\left(i_{j}, k+1\right)
\end{aligned}
$$

where

$$
f(i, j)= \begin{cases}\left.L P_{d_{j}-d_{i}}\left(h_{d_{i}+1}, \ldots, h_{d_{j}}\right) ; q\right) & \text { if } i<j \\ 1 & \text { if } i=j \\ 0 & \text { if } i>j .\end{cases}
$$

Following the discussion of Stanley [14, p.69], we obtain that

Theorem $2.2 \beta_{F}(D, q)$ is the determinant of a $(k+1) \times(k+1)$ matrix with its $(i, j)$ entry $f(i, j+1), 0 \leqslant i, j \leqslant k$. That is,

$$
\beta_{F}(D, q)=\operatorname{det}\left[f_{i, j+1}\right]_{0}^{k}
$$

where $f(i, j)$ is given by $(2.2)$.

When the Ferrers board $F$ is an $n \times n$ square, $h_{i}=n-i+1$, and

$$
L P_{j-i}\left(\left(h_{i+1}, \ldots, h_{j}\right) ; q\right)=\left[\begin{array}{c}
n-i \\
j-i
\end{array}\right] .
$$

Theorems 2.1 and 2.2 reduce to the classical results

$$
\sum_{\substack{\pi \in \mathfrak{E}_{n} \\
D e s(\pi) \subseteq D}} q^{\operatorname{inv}(\pi)}=\left[\begin{array}{c}
n \\
d_{1}, d_{2}-d_{1}, \ldots, n-d_{k}
\end{array}\right]
$$

and

$$
\sum_{\substack{\pi \in \mathfrak{G}_{n} \\
\operatorname{Des}(\pi)=D}} q^{\operatorname{inv}(\pi)}=\operatorname{det}\left[\left[\begin{array}{c}
n-d_{i} \\
d_{j+1}-d_{i}
\end{array}\right]\right]_{0}^{k} .
$$

For a general Ferrers board with $n$ rows and $n$ columns, let's check two extreme cases: $D=\{1,2, \ldots, n-1\}$ and $D=\emptyset$.

- Case 1. $D=\{1,2, \ldots, n-1\}$ : Theorem 2.1 yields the identity

$$
\sum_{\sigma \in F} q^{\operatorname{inv}(\sigma)}=\alpha_{F}(=\{1,2, \ldots, n-1\}, q)=\prod_{i=1}^{n} L P_{1}\left(\left(h_{i}\right) ; q\right)=\prod_{i=1}^{n}\left[h_{i}\right] .
$$

Note that permutation fillings of a Ferrers board with $n$ rows and $n$ columns correspond to complete matchings of $\{1, \ldots, 2 n\}$ with fixed sets of left endpoints and right endpoints, and an inversion of the permutation is exactly a nesting of the 
matching; See de Mier [6] and Kasraoui [10]. To see this, for a given Ferrers board $F$ with $n$ rows and $n$ columns, one traverses the path from the lower-left corner to the top-right corner, and records the path by its steps $a_{1}, a_{2}, \ldots, a_{2 n}$ where $a_{i}=E$ if the $i$ th step is East, and $a_{i}=N$ if the $i$ th step is North. Let $L=\left\{i: a_{i}=E\right\}$ and $R=\left\{i: a_{i}=N\right\}$. Then 01-fillings of $F$ considered here are in one-to-one correspondence with the matchings of $\{1, \ldots, 2 n\}$ for which the set of left endpoints is $L$ and the set of right endpoints is $R$. For example, in the following Ferrers board $F$, traversing from the lower-left corner to the top-right corner, we get the sequence EENENENN. Thus $L=1,2,4,6$ and $R=3,5,7,8$. The filling given in the figure corresponds to the matching $\{(1,7),(2,3),(4,5),(6,8)\}$.

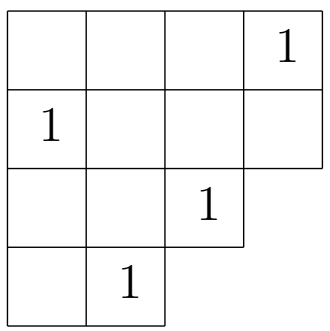

It follows that equation (2.4) is exactly the generating function of the statistic $n e_{2}(M)$, which is the number of nestings in a matching $M$, counted over all the matchings with given sets of left and right endpoints. That is,

$$
\sum_{M} q^{n e_{2}(M)}=\prod_{i=1}^{n}\left[h_{i}\right],
$$

which matches the known results in $[7,10]$.

- Case $2, D=\emptyset$ : We have

$$
\alpha_{F}(\emptyset, q)=\beta_{F}(\emptyset, q)=L P_{n}\left(\left(h_{1}, \ldots, h_{n}\right), q\right)
$$

Note that $h_{n}=1$, hence $L P_{n}\left(\left(h_{1}, \ldots, h_{n}\right), q\right)=1$ iff $r_{i} \geqslant i$ for all $i$, where the only permutation in the Ferrers board $F$ with no descents is the identity permutation; otherwise $\operatorname{Path}_{n}\left(h_{1}, \ldots, h_{n}\right)=\emptyset$ and $L P_{n}\left(\left(h_{1}, \ldots, h_{n}\right), q\right)=0$.

Theorems 2.1 and 2.2 can be used to get a formula for the joint distribution of $\operatorname{des}(\sigma)$ and $\operatorname{inv}(\sigma)$ over permutations in $F$. Let

$$
A_{F}(t, q)=\sum_{\sigma \in F} t^{1+\operatorname{des}(\sigma)} q^{\operatorname{inv}(\sigma)}
$$

Theorem 2.3

$$
A_{F}(t, q)=(1-t)^{n} \operatorname{per}(M)
$$


where $M$ is an $n \times n$ matrix whose $(i, j)$-entry is given by

$$
M_{i j}= \begin{cases}\frac{t}{1-t} L P_{j-i+1}\left(\left(h_{i}, \ldots, h_{j}\right) ; q\right) & \text { if } i \leqslant j \\ 1 & \text { if } i=j+1 \\ 0 & \text { if } i>j+1\end{cases}
$$

and $\operatorname{per}(M)$ is the permanent of the matrix $M$.

Proof. We have

$$
\begin{aligned}
\sum_{\sigma \in F} t^{1+\operatorname{des}(\sigma)} q^{\operatorname{inv}(\sigma)} & =\sum_{D \subseteq\{1,2, \ldots, n-1\}} t^{1+|D|} \beta_{F}(D, q) \\
& =\sum_{D \subseteq\{1,2, \ldots, n-1\}} t^{1+|D|} \sum_{T: T \subseteq D}(-1)^{|D-T|} \alpha_{F}(T, q) \\
& =\sum_{T \subseteq\{1,2, \ldots, n-1\}} \alpha_{F}(T, q) \sum_{D: T \subseteq D}(-1)^{|D-T|} t^{1+|T|+|D-T|} \\
& =(1-t)^{n} \sum_{T=\left\{t_{1}, \ldots, t_{k}\right\}<}\left(\frac{t}{1-t}\right)^{1+k} \Delta_{T}\left(L P_{n}(\mathbf{h})\right) \\
& =(1-t)^{n} \operatorname{per}(M),
\end{aligned}
$$

where $M$ is an $n \times n$ matrix as described in Theorem, and $\Delta_{D}\left(L P_{n}(\mathbf{h})\right)$ denotes the right-hand side of $(2.1)$.

Remark 2.1 We remark that descents of permutations in a Ferrers board provide an example of one-dependent determinantal point processes, as studied by Borodin, Diaconis and Fulman [1]. Let $U$ be a finite set. A point process on $U$ is a probability measure $P$ on the $2^{|U|}$ subsets of $U$. One simple way to specify $P$ is via its correlation functions $\rho(A)$, where for $A \subseteq U$,

$$
\rho(A)=P\{S: S \supseteq A\} .
$$

A point process is determinantal with kernel $K(x, y)$ if

$$
\rho(A)=\operatorname{det}[K(x, y)]_{x, y \in A} .
$$

It is one-dependent if $\rho(X \cup Y)=\rho(X) \rho(Y)$ whenever $\operatorname{dist}(X, Y) \geqslant 2$.

Borodin et al. showed that many examples from combinatorics, algebra and group theory are determinantal one-dependent point processes, for example, the carries process, the descent set of uniformly random permutations, and the descent set in Mallows model [1]. For these three cases, the point processes are stationary, while the descent set of permutations in a Ferrers board corresponds to a determinantal one-dependent point process that is not stationary. Explicitly, for any set $D=\left\{d_{1}, \ldots, d_{k}\right\}$ with $1 \leqslant d_{1}<$ $\cdots<d_{k} \leqslant n-1$, let $P_{F}(D)=\beta_{F}(D) /\left(\prod_{i=1}^{n} h_{i}\right)$. Using [1, Theorem 7.5] we obtain that $P_{F}$ is a determinantal, one-dependent process with correlation functions

$$
\rho(D)=\alpha_{F}(D)=\operatorname{det}\left[K\left(d_{i}, d_{j}\right)\right]_{i, j=1}^{k}
$$


and with correlation kernel

$$
K(x, y)=\delta_{x, y}+\left(E^{-1}\right)_{x, y+1},
$$

where $E$ is the upper triangular matrix $E=[e(i-1, j)]_{i, j=1}^{n}$ whose entries are given by

$$
e(i, j)= \begin{cases}L P_{j-i}\left(h_{i+1}, \ldots, h_{j}\right) & \text { if } i<j \\ 1 & \text { if } i=j \\ 0 & \text { if } i>j\end{cases}
$$

\section{Permutations in the truncated board $n \times n-\Delta_{s}$}

For a general non-decreasing sequence of positive integers $\mathbf{s}, L P_{n}(\mathbf{s}, q)$ can be computed by a determinant formula (see, for example, [11]). But there is no simple closed formula. In the special cases that the Ferrers board $F$ is obtained from truncating the $n \times n$ square board by a triangular board in the corner, we can describe the joint distribution of the statistics $\operatorname{des}(\sigma)$ and $\operatorname{inv}(\sigma)$ by identities of their bi-variate generating functions.

Let $\Delta_{s}$ be the triangular board with row size $(s, s-1, \ldots, 1)$. For $n \geqslant s$, let $\Lambda_{n, s}$ be the truncated board $n \times n-\Delta_{s}$ consisting of cells that are lying in $0 \leqslant x, y \leqslant n$ and above the line $y=x-(n-s)$. In other words, $\Lambda_{n, s}$ is the Ferrers board whose row lengths are $(n-s, n-s+1, \ldots, n, \ldots, n)$. See the following figure for $\Lambda_{n, s}$ with with $n=7$ and $s=4$.

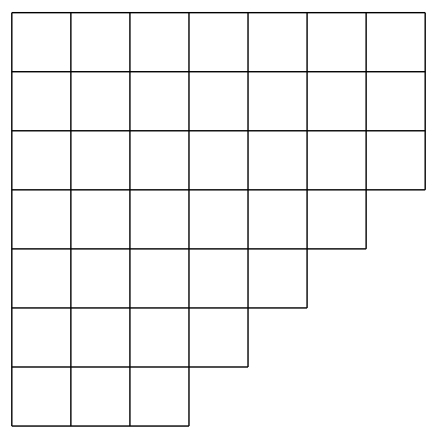

Now let $D=\left\{d_{1}, \ldots, d_{k}\right\}$ with $1 \leqslant d_{1}<\cdots<d_{k} \leqslant n-1$. We shall compute the joint distribution of $1+$ des and inv over all permutations in $\Lambda_{n, s}$ using the formulas obtained in Section 2. Again let $d_{0}=0$ and $d_{k+1}=n$. Let $\delta_{i}=d_{i}-d_{i-1}$ for $i=1, \ldots, k+1$, and assume that $j$ is the particular index to make $d_{j} \leqslant s<d_{j+1}$ occur.

First we compute $\alpha_{\Lambda_{n, s}}(D, q)$. Let $r_{i}$ be the size of row $i$ in $\Lambda_{n, s}$. Then

$$
r_{i}= \begin{cases}n-s-1+i & \text { if } i \leqslant s \\ n & \text { if } s<i \leqslant n .\end{cases}
$$

Let $h_{i}=r_{i}-i+1$. Then

1. For $0 \leqslant i<j$,

$$
L P_{d_{i+1}-d_{i}}\left(\left(h_{d_{i}+1}, \ldots, h_{d_{i+1}}\right), q\right)=L P_{\delta_{i+1}}((n-s, \ldots, n-s), q)=\left[\begin{array}{c}
n-s-1+\delta_{i+1} \\
\delta_{i+1}
\end{array}\right] .
$$


2. For $i=j$,

$$
\begin{aligned}
L P_{d_{j+1}-d_{j}}\left(\left(h_{d_{j}+1}, \ldots, h_{d_{i+1}}\right), q\right) & =L P_{\delta_{j+1}}\left(\left((n-s)^{s-d_{j}}, n-s-1, \ldots, n-d_{j+1}+1\right), q\right) \\
& =\left[\begin{array}{c}
n-d_{j+1}+\delta_{j+1} \\
\delta_{j+1}
\end{array}\right] \\
& =\left[\begin{array}{c}
n-d_{j} \\
\delta_{j+1}
\end{array}\right] .
\end{aligned}
$$

3. For $i>j$,

$$
\begin{aligned}
L P_{d_{i+1}-d_{i}}\left(\left(h_{d_{i}+1}, \ldots, h_{d_{i+1}}\right), q\right) & =L P_{\delta_{i+1}}\left(\left(\left(n-d_{i}, n-d_{i}-1, \ldots, n-d_{i+1}+1\right), q\right)\right. \\
& =\left[\begin{array}{c}
n-d_{i+1}+\delta_{i+1} \\
\delta_{i+1}
\end{array}\right] \\
& =\left[\begin{array}{c}
n-d_{i} \\
\delta_{i+1}
\end{array}\right] .
\end{aligned}
$$

Summing over all permutations $\sigma$ with $\operatorname{Des}(\sigma) \subseteq D$ in the Ferrers board $\Lambda_{n, s}$, we obtain

$$
\begin{aligned}
\alpha_{\Lambda_{n, s}}(D, q)=\sum_{\substack{\sigma \in \Lambda_{n, s} \\
D e s(\sigma) \subseteq D}} q^{\operatorname{inv}(\sigma)} & =\prod_{i=1}^{j}\left[\begin{array}{c}
n-s-1+\delta_{i} \\
\delta_{i}
\end{array}\right] \cdot \prod_{i=j}^{k}\left[\begin{array}{c}
n-d_{i} \\
\delta_{i+1}
\end{array}\right] \\
& =\prod_{i=1}^{j}\left[\begin{array}{c}
n-s-1+\delta_{i} \\
\delta_{i}
\end{array}\right] \cdot\left[\begin{array}{c}
n-d_{j} \\
\Delta\left(D_{j}\right)
\end{array}\right],
\end{aligned}
$$

where $\Delta\left(D_{j}\right)$ represents the sequence $\delta_{j+1}, \ldots, \delta_{k+1}$.

Hence the Principle of Inclusion-Exclusion leads to

$$
\beta_{\Lambda_{n, s}}(I, q)=\sum_{\substack{\sigma \in \Lambda_{n, s} \\
\operatorname{Des}(\sigma)=I}} q^{\operatorname{inv}(\sigma)}=\sum_{D \subseteq I}(-1)^{|I|-|D|}\left[\begin{array}{l}
n-d_{j} \\
\Delta\left(D_{j}\right)
\end{array}\right] \prod_{i=1}^{j}\left[\begin{array}{c}
n-s-1+\delta_{i} \\
\delta_{i}
\end{array}\right] .
$$

Let $F_{n, s}(q, t)$ be the bi-variate generating function of the statistics inv and des over all permutations in the board $\Lambda_{n, s}$. That is,

$$
\begin{aligned}
F_{n, s}(q, t) & =\sum_{\sigma \in \Lambda_{s}} t^{1+\operatorname{des}(\sigma)} q^{\operatorname{inv}(\sigma)} \\
& =\sum_{I \subseteq\{1,2, \ldots, n-1\}} t^{1+|I|} \sum_{\substack{\sigma \in \Lambda_{n}, s \\
D e s(\sigma)=I}} q^{\operatorname{inv}(\sigma)} .
\end{aligned}
$$

Let $(a ; q)_{n}=(1-a)(1-a q) \cdots\left(1-a q^{n-1}\right)$ and $(a, q)_{\infty}=\prod_{i=0}^{\infty}\left(1-a q^{i}\right)$. Our main result here is an analog of the formula (1.7). Explicitly, we show that $F_{n, s}(q, t)$ can be expressed as a linear combination of $s+1$ terms, each of which satisfies a $q$-identity similar to $(1.7)$. 
Theorem 3.1 For $n \leqslant s, F_{n, s}(q, t)=0$. For $n>s$, we have

$$
F_{n, s}(q, t)=\theta_{0} F_{n, s}^{(0)}(q, t)+\theta_{1} F_{n, s}^{(1)}(q, t)+\cdots+\theta_{s} F_{n, s}^{(s)}(q, t),
$$

where the $\theta_{k}$ 's are defined by the formal power series

$$
\sum_{k=0}^{\infty} \theta_{k} z^{k}=\left(1-\frac{t}{1-t}\left(\frac{1}{(z ; q)_{n-s}}-1\right)\right)^{-1}
$$

and for each $i=0,1, \ldots, s$, the term $F_{n, s}^{(i)}(q, t)$ is given by the identity

$$
\sum_{n \geqslant s+1} \frac{z^{n}}{[n-i] !} \frac{F_{n, s}^{(i)}(q, t)}{(1-t)^{n}}=\frac{\frac{t}{1-t} \sum_{k \geqslant s+1-i} \frac{z^{k}}{[k] !}}{1-\frac{t}{1-t} \sum_{k \geqslant 1} \frac{z^{k}}{[k] !}}
$$

Proof. As before assume $D=\left\{d_{1}, d_{2}, \ldots, d_{k}\right\}$ with $d_{0}=0$ and $d_{k+1}=n$. Let $j$ be the index uniquely decided by $d_{j} \leqslant s<d_{j+1}$. For $n \geqslant s+1$, by the equation (3.1) we have

$$
\begin{aligned}
F_{n, s}(q, t) & =\sum_{I \subseteq\{1,2, \ldots, n-1\}} t^{1+|I|} \sum_{D \subseteq I}(-1)^{|I|-|D|}\left[\begin{array}{l}
n-d_{j} \\
\Delta\left(D_{j}\right)
\end{array}\right] \prod_{i=1}^{j}\left[\begin{array}{c}
n-s-1+\delta_{i} \\
\delta_{i}
\end{array}\right] \\
& =\sum_{D \subseteq\{1,2, \ldots, n-1\}}\left[\begin{array}{l}
n-d_{j} \\
\Delta\left(D_{j}\right)
\end{array}\right] \prod_{i=1}^{j}\left[\begin{array}{c}
n-s-1+\delta_{i} \\
\delta_{i}
\end{array}\right] \sum_{I: D \subseteq I}(-1)^{|I|-|D|} t^{1+|I|} \\
& =\sum_{D \subseteq\{1,2, \ldots, n-1\}}\left[\begin{array}{c}
n-d_{j} \\
\Delta\left(D_{j}\right)
\end{array}\right] \prod_{i=1}^{j}\left[\begin{array}{c}
n-s-1+\delta_{i} \\
\delta_{i}
\end{array}\right](1-t)^{n-1-|D|} t^{1+|D|} \\
& =\sum_{k=0}^{n-1} t^{1+k}(1-t)^{n-1-k} \sum_{\substack{\delta_{1}+\delta_{2}+\ldots+\delta_{k+1}=n \\
\delta_{1}+\ldots+\delta_{j} \leqslant s<\delta_{1}+\ldots+\delta_{j+1}}} \frac{\left[n-d_{j}\right] ! \prod_{i=1}^{j}\left[n-s-1+\delta_{i}\right] !}{\left[\delta_{1}\right] ! \cdots\left[\delta_{k+1}\right] !([n-s-1] !)^{j}} .
\end{aligned}
$$

Let $d_{i}=l$ and $\gamma=\frac{t}{1-t}$. Then,

$$
\begin{aligned}
& \frac{F_{n, s}(q, t)}{(1-t)^{n}} \\
& =\sum_{l=0}^{s} \sum_{k \geqslant 0} \gamma^{1+k} \sum_{\substack{\delta_{1}+\delta_{2}+\ldots+\delta_{k+1}=n \\
l=\delta_{1}+\ldots+\delta_{j} \leqslant s<\delta_{1}+\ldots+\delta_{j+1}}} \frac{[n-l] ! \prod_{i=1}^{j}\left[n-s-1+\delta_{i}\right] !}{\left[\delta_{1}\right] ! \cdots\left[\delta_{k+1}\right] !([n-s-1] !)^{j}} . \\
& =\sum_{l=0}^{s}\left(\sum_{\substack{j \\
\delta_{1}+\ldots+\delta_{j}=l}} \gamma^{j} \prod_{i=1}^{j}\left[\begin{array}{c}
n-s-1+\delta_{i} \\
\delta_{i}
\end{array}\right]\right)\left(\sum_{\substack{k ; \delta_{j+1} \geqslant s+1-l \\
\delta_{j+1}+\delta_{j+2}+\ldots+\delta_{k+1}=n-l}} \frac{\gamma^{k+1-j}[n-l] !}{\left[\delta_{j+1}\right] ! \cdots\left[\delta_{k+1}\right] !}\right)
\end{aligned}
$$


Let $\theta_{0}=1$ and for $l=1, \ldots, s$,

$$
\theta_{l}:=\sum_{\substack{j \\
\delta_{1}+\ldots+\delta_{j}=l}} \gamma^{j} \prod_{i=1}^{j}\left[\begin{array}{c}
n-s-1+\delta_{i} \\
\delta_{i}
\end{array}\right]
$$

and

$$
F_{n, s}^{(l)}:=(1-t)^{n} \sum_{\substack{k ; \tau_{0} \geqslant s+1-l \\ \tau_{0}+\tau_{1}+\cdots+\tau_{k}=n-l}} \frac{\gamma^{k+1}[n-l] !}{\left[\tau_{0}\right] ! \cdots\left[\tau_{k}\right] !} .
$$

Then

$$
F_{n, s}(q, t)=\theta_{0} F_{n, s}^{(0)}(q, t)+\theta_{1} F_{n, s}^{(1)}(q, t)+\cdots+\theta_{s} F_{n, s}^{(s)}(q, t)
$$

We show that $\theta_{l}$ and $F_{n, s}^{(l)}(q, t)$ satisfy (3.3) and (3.4).

First, observe that for $l>0, \theta_{l}$ is the coefficient of $z^{l}$ in the formal power series

$$
\sum_{j=0}^{\infty}\left(\gamma \sum_{k=1}^{\infty}\left[\begin{array}{c}
n-s-1+k \\
k
\end{array}\right] z^{k}\right)^{j}
$$

Using the $q$-analog of the binomial theorem

$$
\sum_{k=0}^{\infty} \frac{(a ; q)_{k}}{(q ; q)_{k}} z^{k}=\frac{(a z ; q)_{\infty}}{(z ; q)_{\infty}}
$$

we have

$$
\begin{aligned}
\sum_{l=0}^{\infty} \theta_{l} z^{l} & =\sum_{j=0}^{\infty}\left(\gamma \sum_{k=1}^{\infty} \frac{[n-s+k-1][n-s+k-2] \cdots[n-s]}{[k] !} z^{k}\right)^{j} \\
& =\sum_{j=0}^{\infty}\left(\gamma \cdot\left(\frac{\left(q^{n-s} z ; q\right)_{\infty}}{(z ; q)_{\infty}}-1\right)\right)^{j} \\
& =\left(1-\gamma\left(\frac{1}{(z ; q)_{n-s}}-1\right)\right)^{-1} .
\end{aligned}
$$

This proves the formula (3.3).

To get the formula (3.4), observe that (3.6) can be written as

$$
\frac{1}{[n-l] !} \frac{F_{n, s}^{(l)}}{(1-t)^{n}}=\left[z^{n-l}\right]\left(\gamma \sum_{\tau_{0} \geqslant s+1-l} \frac{z^{\tau_{0}}}{\left[\tau_{0}\right] !} \cdot \sum_{k=0}^{\infty} \gamma^{k}\left(\sum_{\tau=1}^{\infty} \frac{z^{\tau}}{[\tau] !}\right)^{k}\right) .
$$


This leads to the identity

$$
\sum_{n \geqslant s+1} \frac{z^{n-l}}{[n-l] !} \frac{F_{n, s}^{(l)}(q, t)}{(1-t)^{n}}=\frac{\frac{t}{1-t} \sum_{k \geqslant s+1-l} \frac{z^{k}}{[k] !}}{1-\frac{t}{1-t} \sum_{k \geqslant 1} \frac{z^{k}}{[k] !}} .
$$

In the case that $s=0, F_{n, s}(q, t)=F_{n, s}^{(0)}(q, t)=A_{n}(t, q)$, and equation (3.4) reduces to the well-known identity (1.7) by letting $u=\frac{z}{1-t}$.

\section{Permutations with bounded drop or excedance size}

Permutations with bounded drop size is related to the bubble sort and sequences that can be translated into juggling patterns [5], whose enumeration was first studied by Chung, Claesson, Dukes, and Graham [4]. For a permutation $\sigma$, we say that $i$ is a drop of $\sigma$ if $\sigma_{i}<i$ and the drop size is $i-\sigma_{i}$. Similarly, we say that $i$ is an excedance of $\sigma$ if $\sigma_{i}>i$, and the excedance size is $\sigma_{i}-i$. It is well-known that the number of excedances is an Eulerian statistic, i.e., has the same distribution as des over the set of permutations.

Following [4], we use $\operatorname{maxdrop}(\sigma)$ to denote the maximum drop of $\sigma$,

$$
\operatorname{maxdrop}(\sigma):=\max \left\{i-\sigma_{i} \mid 1 \leqslant i \leqslant n\right\},
$$

and similarly, $\operatorname{maxexc}(\sigma)$ to denote the maximum excedance size of $\sigma$,

$$
\operatorname{maxexc}(\sigma):=\max \left\{\sigma_{i}-i \mid 1 \leqslant i \leqslant n\right\} .
$$

Let $\mathcal{B}_{n, k}=\left\{\sigma \in \mathfrak{S}_{n} \mid \operatorname{maxdrop}(\sigma) \leqslant k\right\}$. It is easy to see that $\left|\mathcal{B}_{n, k}\right|=k !(k+1)^{n-k}$ : Just note that there are $(k+1)^{n-k}$ ways to determine $\sigma_{n}, \cdots, \sigma_{k+1}$ in the correct order, one after another, and the remaining is clear (e.g., see [5, Thm.1]). In [4], Chung et al. defined the $k$-maxdrop descent polynomials

$$
B_{n, k}(t):=\sum_{\sigma \in \mathcal{B}_{n, k}} t^{\operatorname{des}(\sigma)}
$$

and obtained recurrences as well as a formula for the generating function $B_{k}(t, z):=$ $\sum_{n \geqslant 0} B_{n, k}(t) z^{n}$.

In this section, we will use the analysis in the previous section to derive a variant generating function for $B_{k}(t, z)$. Explicitly, we get an exact formula for

$$
E_{k}(t, z):=\sum_{n \geqslant k} B_{n, k}(t) z^{n}=\sum_{n \geqslant k}\left(\sum_{\sigma \in \mathcal{B}_{n, k}} t^{d e s(\sigma)}\right) z^{n} .
$$


First, let $\mathcal{B}_{n, k}^{\prime}=\left\{\sigma \in \mathfrak{S}_{n} \mid \operatorname{maxexc}(\sigma) \leqslant k\right\}$. It is clear that the map $a_{1} a_{2} \ldots a_{n} \mapsto$ $\left(n+1-a_{n}\right)\left(n+1-a_{n-1}\right) \ldots\left(n+1-a_{1}\right)$ is a bijection from $\mathcal{B}_{n, k}$ to $\mathcal{B}_{n, k}^{\prime}$ that preserves the statistic $\operatorname{des}(\sigma)$ and $\operatorname{inv}(\sigma)$. It follows that

$$
B_{n, k}(t)=\sum_{\sigma \in \mathcal{B}_{n, s}^{\prime}} t^{\operatorname{des}(\sigma)} \quad \text { and hence } \quad E_{k}(t, z)=\sum_{n \geqslant k}\left(\sum_{\sigma \in \mathcal{B}_{n, k}^{\prime}} t^{\operatorname{des}(\sigma)}\right) z^{n} .
$$

Note that $\mathcal{B}_{n, k}^{\prime}$ is the set of permutations $\sigma \in \mathfrak{S}_{n}$ satisfying $\sigma_{i} \leqslant i+k$. It is easy to check that it is exactly the set of permutations on the truncated board $\Lambda_{n, n-k-1}$. Hence for $n \geqslant k+1$, we have

$$
\sum_{\sigma \in \mathcal{B}_{n, k}} t^{1+\operatorname{des}(\sigma)} q^{\operatorname{inv}(\sigma)}=F_{n, n-k-1}(q, t)
$$

and Theorem 3.1 with $s=n-k-1$ gives a description of $F_{n, n-k-1}(q, t)$.

To obtain the ordinary generating function for $B_{n, k}(t)$, set $q=1$. As before, let $\gamma=t /(1-t)$. Then formula (3.5) becomes the following equation for $n \geqslant k+1$ :

$$
\frac{t B_{n, k}(t)}{(1-t)^{n}}=\sum_{l=0}^{n-k}\left(\sum_{\substack{j \\
\delta_{1}+\ldots+\delta_{j}=l}} \gamma^{j} \prod_{i=1}^{j}\left(\begin{array}{c}
k+\delta_{i} \\
\delta_{i}
\end{array}\right)\right)\left(\sum_{\substack{p ; \delta_{j+1}>n-k-l \\
\delta_{j+1}+\delta_{j+2}+\ldots+\delta_{p+1}=n-l}} \frac{\gamma^{p+1-j}(n-l) !}{\delta_{j+1} ! \cdots \delta_{k+1} !}\right) .
$$

(Note that from the analysis, the upper limit of $l$ can include $n-k$.)

Let $\theta_{0}^{(k)}=1$ and for $l \geqslant 1$,

$$
\theta_{l}^{(k)}:=\sum_{\substack{j ; \delta_{1}+\ldots+\delta_{j}=l \\
\delta_{i}>0}} \gamma^{j} \prod_{i=1}^{j}\left(\begin{array}{c}
k+\delta_{i} \\
\delta_{i}
\end{array}\right)
$$

and

$$
c_{n-l}^{(k)}:=\sum_{\tau_{0}>n-k-l} \gamma\left(\begin{array}{c}
n-l \\
\tau_{0}
\end{array}\right) \cdot \sum_{\substack{\tau_{1}+\cdots+\tau_{p}=n-l-\tau_{0} \\
\tau_{i} \geqslant 1}} \gamma^{p}\left(\begin{array}{c}
n-l-\tau_{0} \\
\tau_{1}, \ldots, \tau_{p}
\end{array}\right), \quad \text { for } k>0 .
$$

For $k=0$, let $c_{n}^{(0)}=\delta_{n, 0}$. Then for any fixed $k \geqslant 0$ and $n \geqslant k+1$, we have

$$
\frac{t B_{n, k}(t)}{(1-t)^{n}}=\sum_{l=0}^{n-k} \theta_{l}^{(k)} c_{n-l}^{(k)}
$$

Letting $q=1$ and $k=n-s-1$ in equation (3.3), we obtain

$$
\Theta_{k}(z)=\sum_{n \geqslant 0} \theta_{n}^{(k)} z^{n}=\left(1-\frac{t}{1-t}\left(\frac{1}{(1-z)^{k+1}}-1\right)\right)^{-1} .
$$


For the coefficient $c_{i}^{(k)}$, using formula (3.5) with $s=0$, we get that

$$
\sum_{\substack{\tau_{1}+\cdots+\tau_{p}=n \\
\tau_{i} \geqslant 1}} \gamma^{p}\left(\begin{array}{c}
n \\
\tau_{1}, \ldots, \tau_{p}
\end{array}\right)=\frac{A_{n}(t)}{(1-t)^{n}}
$$

where $A_{n}(t)$ is the classical Eulerian polynomial defined by

$$
A_{n}(t)=\sum_{\sigma \in \mathfrak{S}_{n}} t^{1+\operatorname{des}(\sigma)}, \quad n>0 .
$$

By convention, we set $A_{0}(t)=1$. It follows that for $k>0$,

$$
c_{n}^{(k)}=\gamma \sum_{p>n-k}\left(\begin{array}{l}
n \\
p
\end{array}\right) \frac{A_{n-p}(t)}{(1-t)^{n-p}}=\gamma \sum_{p=0}^{k-1}\left(\begin{array}{l}
n \\
p
\end{array}\right) \frac{A_{p}(t)}{(1-t)^{p}} .
$$

Writing as a generating function, we obtain that for $k>0$,

$$
C_{k}(z)=\sum_{n \geqslant k} c_{n}^{(k)} z^{n}=\gamma \sum_{p=0}^{k-1} \frac{A_{p}(t)}{(1-t)^{p}} \sum_{n \geqslant k}\left(\begin{array}{l}
n \\
p
\end{array}\right) z^{n}
$$

which leads to

$$
C_{k}(z)=\frac{t}{1-t} \sum_{p=0}^{k-1} \frac{A_{p}(t)}{(1-t)^{p}}\left(\frac{z^{p}}{(1-z)^{p+1}}-\sum_{n=p}^{k-1}\left(\begin{array}{l}
n \\
p
\end{array}\right) z^{n}\right) .
$$

For $k=0, C_{0}(z)=1$.

Observe that equation (4.2) is true for $n=k$ as well. In fact it is equivalent to the identity

$$
A_{k}(t)=t \sum_{p=0}^{k-1}\left(\begin{array}{l}
k \\
p
\end{array}\right)(1-t)^{k-p-1} A_{p}(t)
$$

which can be readily checked by using the following expression of the Eulerian polynomial, see, for example [3, Lemma 14.1, p.517],

$$
A_{n}(t)=(1-t)^{n+1} \sum_{j=0}^{\infty} j^{n} t^{j}, \quad n \geqslant 0 .
$$

Therefore for all $n \geqslant k$,

$$
B_{n, k}(t)=\frac{(1-t)^{n}}{t} \sum_{l=0}^{n-k} \theta_{l}^{(k)} c_{n-l}^{(k)}
$$

Multiplying both sides by $z^{n}$ and summing over $n \geqslant k$, we have obtained the generating function $E_{k}(t, z)$. 
Theorem 4.1 Let

$$
E_{k}(t, z)=\sum_{n \geqslant k} B_{n, k}(t) z^{n}=\sum_{n \geqslant k}\left(\sum_{\sigma \in \mathcal{B}_{n, k}} t^{\operatorname{des}(\sigma)}\right) z^{n} .
$$

Then $E_{0}(t, z)=1 /(1-z)$ and for $k \geqslant 1$,

$$
E_{k}(t, z)=\frac{1}{t} \Theta_{k}((1-t) z) C_{k}((1-t) z)
$$

where $\Theta_{k}(z)$ and $C_{k}(z)$ are given in formulas (4.3) and (4.5). Explicitly,

$$
E_{k}(t, z)=\frac{\sum_{p=0}^{k-1} A_{p}(t)\left(\frac{z^{p}}{(1-(1-t) z)^{p+1}}-\sum_{n=p}^{k-1}\left(\begin{array}{l}
n \\
p
\end{array}\right)(1-t)^{n-p} z^{n}\right)}{1-\frac{t}{(1-(1-t) z)^{k+1}}} .
$$

Example 4.1 For the case $k=1$, formula (4.7) gives

$$
E_{1}(t, z)=\frac{\frac{1}{1-(1-t) z}-1}{1-\frac{t}{(1-(1-t) z)^{2}}}=\frac{z(1-(1-t) z)}{1-z(2-(1-t) z)} .
$$

Comparing with equation (5) in [4], and noting that the summation of $B_{k}(z, y)$ in [4] starts from $n=0$, one checks easily that the two formulas agree with each other.

Acknowledgement The authors wish to thank an anonymous referee for the very careful reading and many helpful suggestions.

\section{References}

[1] Alexei Borodin, Persi Diaconis, and Jason Fulman. On adding a list of numbers (and other one-dependent determinantal processes). Bull. Amer. Math. Soc. (N.S.), 47(4):639-670, 2010.

[2] Leonard Carlitz and John Riordan. Two element lattice permutation numbers and their q-generalization. Duke Math. J., 31:371-388, 1964.

[3] Charalambos A. Charalambides. Enumerative combinatorics. CRC Press Series on Discrete Mathematics and its Applications. Chapman \& Hall/CRC, Boca Raton, FL, 2002.

[4] Fan Chung, Anders Claesson, Mark Dukes, and Ronald Graham. Descent polynomials for permutations with bounded drop size. European J. Combin., 31(7):1853-1867, 2010.

[5] Fan Chung and Ron Graham. Primitive juggling sequences. Amer. Math. Monthly, 115(3):185-194, 2008. 
[6] Anna de Mier. On the symmetry of the distribution of $k$-crossings and $k$-nestings in graphs. Electron. J. Combin., 13(1):Note 21, 6 pp. (electronic), 2006.

[7] M. de Sainte-Catherine. Couplages et pfaffiens en combinatoire, physique et informatique. Master's thesis, University of Bordeaux I.

[8] Adriano M. Garsia. On the "maj" and "inv" $q$-analogues of Eulerian polynomials. Linear and Multilinear Algebra, 8(1):21-34, 1979/80.

[9] Ira M. Gessel. Generating functions and enumeration of sequences. PhD thesis, M.I.T., 1977.

[10] Anisse Kasraoui. Ascents and descents in 01-fillings of moon polyominoes. European J. Combin., 31(1):87-105, 2010.

[11] Sri Gopal Mohanty. Lattice path counting and applications. Academic Press [Harcourt Brace Jovanovich Publishers], New York, 1979. Probability and Mathematical Statistics.

[12] John Riordan. An introduction to combinatorial analysis. Wiley Publications in Mathematical Statistics. John Wiley \& Sons Inc., New York, 1958.

[13] Richard P. Stanley. Binomial posets, Möbius inversion, and permutation enumeration. J. Combinatorial Theory Ser. A, 20(3):336-356, 1976.

[14] Richard P. Stanley. Enumerative combinatorics. Vol. 1, volume 49 of Cambridge Studies in Advanced Mathematics. Cambridge University Press, Cambridge, 1997. With a foreword by Gian-Carlo Rota, Corrected reprint of the 1986 original. 